\title{
Composite Higgs phenomenology
}

\section{Gautam Bhattacharyya*t}

Saha Institute of Nuclear Physics, HBNI, 1/AF Bidhan Nagar, Kolkata 700064, India

E-mail: gautam.bhattacharyya@saha.ac.in

Is the Higgs boson elementary or composite? With increasingly precise measurements of the Higgs couplings to other Standard Model particles, this question can be answered quantitatively with reasonable assumptions and approximations. Here I shall discuss the minimal and nonminimal versions of composite Higgs models by briefly introducing the concept behind constructing such models with a phenomenological outlook, highlighting the main experimental constraints on the model parameters. Mention will be made of fine-tuning involved in constructing a realistic composite Higgs model.

Corfu Summer Institute 2018 "School and Workshops on Elementary Particle Physics and Gravity" (CORFU2018)

31 August - 28 September, 2018

Corfu, Greece

${ }^{*}$ Speaker.

${ }^{\dagger}$ Work done in collaboration with Avik Banerjee, Nilanjana Kumar and Tirtha Sankar Ray. 


\section{Introduction}

Composite Higgs scenario was introduced to address the naturalness problem [1,2]. As is clear from the word 'composite', instead of considering the Higgs boson as a point particle, in this setup it is conceived as an extended object with geometric size $l_{h}^{-1}=m_{*}=$ few TeV. Here, the Higgs boson is a pseudo Nambu-Goldstone boson (pNGB) arising from spontaneous breaking of some global symmetry by exotic fermion condensation at that scale $[3,4,5]$. Explicit symmetry breaking by Yukawa and gauge interactions is responsible for creating its potential and generation of its mass. The whole mechanism is reminiscent of the pion mass generation in QCD via chiral symmetry breaking. The idea behind composite Higgs model building started with lessons taken from the failure of technicolor theories as an explanation of electroweak breaking. Technicolor of course did not need the Higgs boson, where some strong coupling after 'slow running' from a UV scale creates the $\mathrm{TeV}$ scale as

$$
m_{*}^{2} \sim m_{\mathrm{UV}}^{2} \exp \left(-16 \pi^{2} / g_{\mathrm{UV}}^{2}\right) .
$$

But even before the discovery of the Higgs boson, technicolor suffered a serious setback from the precision measurement of the oblique electroweak $S$ parameter where it leaves an unacceptably large contribution. From an effective field theory point of view, the relevant dimension- 6 operator contributing to the $S$ parameter is given by

$$
\mathscr{O}=\frac{1}{f^{2}} H^{\dagger} W_{\mu v} B^{\mu v} H
$$

where $H$ is the Higgs doublet with $v=246 \mathrm{GeV}$ as its vacuum expectation value (vev), $f$ is the decay constant, analogous to that of pion, but associated with some high scale QCD-like theory. Technicolor-like situation is emulated where the strong condensation scale $f \sim v$, which leads to $S \sim v^{2} / f^{2} \sim 1$. This is in clear conflict with electroweak precision measurements which yield $S \sim$ 0.01. In the present context, pure technicolor is ruled out just by the discovery of the Higgs boson. Here comes the utility of composite Higgs scenario which captures many features of technicolor but it is not quite the same theory. Here the role of strong dynamics is to produce a set of NambuGoldstone bosons through spontaneous breaking of some global symmetry, and once those degrees of freedom are available, explicit breaking by gauge / Yukawa interaction would create an effective potential involving those NGBs. Those NGBs thus become pseudo-NGBs - one of them turns out to be the Higgs boson.

Before we get into a specific NGB framework, we first note that in such theories the Higgs boson couples non-linearly with gauge bosons and fermions. More specifically, not only dimension- 4 but also higher dimensional terms involving increasingly higher powers of the Higgs field appear in the effective Lagrangian [6]. For illustration, we start with a Lagrangian whose kinetic part contains dimension- 4 and dimension- 6 terms:

$$
\mathscr{L}_{\text {kin }}=\left|\partial_{\mu} H\right|^{2}+\frac{c_{H}}{2 f^{2}}\left|\partial_{\mu}\left(H^{\dagger} H\right)\right|^{2} .
$$

The gauge part, however, contains only the standard dimension- 4 terms as

$$
\mathscr{L}_{\text {gauge }}=\frac{g^{2}}{2}\left(H^{\dagger} H\right)\left(W_{\mu} W^{\mu}+\frac{1}{2 \cos ^{2} \theta_{W}} Z_{\mu} Z^{\mu}\right),
$$


where adding a dimension- 6 piece with another $\left(H^{\dagger} H\right)$ factor would not cause any physical change as the effect can be absorbed in the redefinition of parameters in the relation $M_{W}=g v / 2$. Now if we canonically normalize the kinetic term in terms of the Higgs field as

$$
\mathscr{L}_{\text {kin }}^{\text {can }}=\left|\partial_{\mu} h_{125}\right|^{2}, \quad \text { where } \quad h_{125} \equiv h \sqrt{1+c_{H} \xi}
$$

with $\xi=v^{2} / f^{2}$, assuming $c_{H}$ to be a small coefficient, the gauge coupling of the Higgs is modified with respect to the corresponding Standard Model (SM) value as

$$
g_{V V h} \simeq g_{V V h}^{\mathrm{SM}} \sqrt{1-c_{H} \xi} .
$$

In the same spirit we write an effective Yukawa Lagrangian with both dimension- 4 and dimension- 6 terms as

$$
\mathscr{L}_{Y u k}=-Y_{f}^{\mathrm{SM}} \bar{Q}_{L} H u_{R}-\Delta\left(\frac{H^{\dagger} H}{f^{2}}\right) Y_{f}^{\mathrm{SM}} \bar{Q}_{L} H u_{R},
$$

where $\Delta$ is a small coefficient. If we extract from here an Yukawa Lagnragian involving the canonically normalized Higgs coupled to the top quark as

$$
\mathscr{L}_{Y u k}^{t}=-Y_{f} \bar{t}_{L} t_{R} h_{125}+\text { h.c. },
$$

then the modified Yukawa coupling turns out to be

$$
Y_{f} \simeq Y_{f}^{\mathrm{SM}}\left[1+\left(\Delta-\frac{c_{H}}{2}\right) \xi\right] .
$$

The above modifications capture a substantial essence of what comes out of a large class of composite Higgs models ultimately in the stage of effective Lagrangian to be used for doing computation. Two important observations are the following.

- The value of $c_{H}$ is fixed to be unity for composite Higgs model associated with $\mathrm{SO}(\mathrm{N}) \rightarrow$ $\mathrm{SO}(\mathrm{N}-1)$ breaking for any $\mathrm{N}$.

- The value of $\Delta$ depends on the choice of fermion representations for a given $\mathrm{SO}(\mathrm{N})$.

\section{Minimal composite Higgs models}

The phenomenologically viable minimal composite Higgs model comprises of $\mathrm{SO}(5) / \mathrm{SO}(4)$ coset [9]. The pNGBs in the coset can be parametrized as

$$
\Sigma=e^{i \frac{\sqrt{2}}{f} \pi_{i}(x) \hat{T}^{i}} \Sigma_{0}=\left(\frac{\pi_{i}}{\pi} \sin \frac{\pi}{f}, \cos \frac{\pi}{f}\right)^{T},
$$

where $\Sigma_{0}=(0,0,0,0, f)^{T}$ is the $\mathrm{SO}(4)$ invariant vacuum and $\pi=\sqrt{\sum_{i} \pi_{i}^{2}}$. In the unitary gauge one can choose $\pi_{1}=\pi_{2}=\pi_{3}=0$ and $\pi_{4}=h$, which leads to $\Sigma=\left(0,0,0, s_{h}, c_{h}\right)^{T}$, with $s_{h}=\sin (h / f)$. The kinetic term for the pNGBs can be constructed using $\Sigma$ as

$$
\mathscr{L}_{\text {kin }}=\frac{1}{2}|\partial \Sigma|^{2}=\frac{1}{2}|\partial h|^{2}\left(1-\frac{h^{2}}{f^{2}}\right)^{-1} .
$$


Canonical kinetic term can be obtained by redefining $h$ as given in Eq. (1.5) with $c_{H}=1$. The $\mathrm{SU}(2)_{\mathrm{L}} \times \mathrm{U}(1)_{\mathrm{Y}}$ subgroup of the unbroken $\mathrm{SO}(4)$ is gauged by introducing $W_{\mu}^{ \pm}$and $B_{\mu}$ gauge bosons. Modification of the gauge couplings of the Higgs follows Eq. (1.6) with $c_{H}=1$. Note that the gauge and Yukawa interactions of the NGBs break $\mathrm{SO}(5)$ explicitly and tangle the pNGB Higgs in a radiatively generated potential.

Within the partial compositeness paradigm, the Yukawa couplings are generated by linear mixing of the SM fermions with some strong sector operators. This implies that after the condensation of the strong sector, the elementary fermions mix with composite resonances. To assign the correct hypercharge of the SM fermions an additional unbroken $\mathrm{U}(1)_{\mathrm{X}}$ is introduced. We will consider only the embeddings of the third generation quarks for the purpose of this paper; however, the same can be extended for other quarks and leptons trivially. The spinorial 4 of $\mathrm{SO}(5)$ is not suitable to embed the top quark due to unacceptably large contribution to $Z b \bar{b}$ vertex, which arises because of the absence of a $(\mathbf{2}, 2)$ in the decomposition of 4 under $\mathrm{SU}(2)_{\mathrm{L}} \times \mathrm{SU}(2)_{\mathrm{R}}[7,8]$. The minimal representation to embed the top quark is the fundamental 5 of $\mathrm{SO}(5)$ with $X=2 / 3$. Decomposition of $5_{2 / 3}$ of $\mathrm{SO}(5) \times \mathrm{U}(1)_{\mathrm{X}}$ under the $\mathrm{SM}$ gauge group is shown below:

$$
\mathbf{5}_{2 / 3} \rightarrow 2_{7 / 6} \oplus 2_{1 / 6} \oplus 1_{2 / 3} .
$$

The relevant incomplete multiplets involving left- and right-handed top quarks in the so called $\mathrm{MCHM}_{5_{\mathrm{L}}-5_{\mathrm{R}}}$ model are given by,

$$
Q_{L}^{5}=\frac{1}{\sqrt{2}}\left(-i b_{L},-b_{L},-i t_{L}, t_{L}, 0\right)^{T}, \quad T_{R}^{5}=\left(0,0,0,0, t_{R}\right)^{T} .
$$

Effective low energy Lagrangian can be constructed employing $\mathrm{SO}(5)$ invariant terms with $\Sigma$ insertions. In $\mathrm{MCHM}_{5_{\mathrm{L}}-5_{\mathrm{R}}}$ model only one invariant term can be formed. The low energy Yukawa Lagrangian is given by

$$
\mathscr{L}_{\text {Yuk }}=\Pi_{L R}\left(q^{2}\right)\left(\bar{Q}_{L}^{5} \cdot \Sigma\right)\left(\Sigma^{T} \cdot T_{R}^{5}\right)+\text { h.c. } \quad \Rightarrow \quad \mathscr{L}_{\text {Yuk }}=\Pi_{L R}\left(q^{2}\right) s_{h} c_{h} \bar{t}_{L} t_{R}+\text { h.c. },
$$

where $\Pi_{L R}\left(q^{2}\right)$ denotes the momentum dependent form factor encoding the strong dynamics. It can be parametrized in terms of the masses and decay constants of the composite resonances (which in the dual 5D theory represent Kaluza-Klein states) as follows:

$$
\Pi_{L R}\left(q^{2}\right)=\sum_{n} \frac{F_{L} F_{R}^{*} m_{n}}{q^{2}+m_{n}^{2}}
$$

For the purpose of low energy phenomenology, one can approximate the form factors with a constant value $\left(\Pi_{L R}\left(q^{2}=0\right)\right)$. After the electroweak symmetry breaking Eq. (2.5) takes the form

$$
\mathscr{L}_{\text {Yuk }}=\left[m_{t}+\left(\frac{m_{t}}{v}\right) \frac{1-2 \xi}{\sqrt{1-\xi}} h_{125}\right] \bar{t}_{L} t_{R}+\text { h.c. },
$$

where we have absorbed $\Pi_{L R}(0)$ in the definition of top mass $m_{t} \simeq \Pi_{L R}(0) \sqrt{\xi(1-\xi)}$. Comparing with Eq. (1.9) and using $c_{H}=1$, we get $\Delta=-1$.

The story, however, completely changes if more than one Yukawa invariants can be constructed, where the form factors cannot be totally absorbed in the definition of $m_{t}$. Such is the 
case when the left- and right-handed top quarks are embedded in the rank-2 symmetric 14 representation of $\mathrm{SO}(5)$ (dubbed as $\mathrm{MCHM}_{14_{\mathrm{L}}-14_{\mathrm{R}}}$ model). Decomposition of 14 under the SM gauge group, and incomplete $\mathrm{SO}(5)$ multiplets containing $t_{L}$ and $t_{R}$ are given by

$$
\mathbf{1 4}_{2 / 3} \rightarrow 3_{5 / 3} \oplus 3_{2 / 3} \oplus 3_{-1 / 3} \oplus 2_{7 / 6} \oplus 2_{1 / 6} \oplus 1_{2 / 3},
$$

and

$$
Q_{L}^{14}=\frac{1}{\sqrt{2}}\left(\begin{array}{ccccc}
0 & 0 & 0 & 0 & -i b_{L} \\
0 & 0 & 0 & 0 & -b_{L} \\
0 & 0 & 0 & 0 & -i t_{L} \\
0 & 0 & 0 & 0 & t_{L} \\
-i b_{L} & -b_{L} & -i t_{L} & t_{L} & 0
\end{array}\right), \quad T_{R}^{14}=\frac{1}{2 \sqrt{5}}\left(\begin{array}{ccccc}
t_{R} & 0 & 0 & 0 & 0 \\
0 & t_{R} & 0 & 0 & 0 \\
0 & 0 & t_{R} & 0 & 0 \\
0 & 0 & 0 & t_{R} & 0 \\
0 & 0 & 0 & 0 & -4 t_{R}
\end{array}\right) .
$$

The Yukawa Lagrangian in this case, containing two invariants can be written as

$$
\begin{aligned}
\mathscr{L}_{\text {Yuk }} & =\Pi_{L R}^{(1)}\left(\Sigma^{T} \cdot \bar{Q}_{L}^{14} \cdot T_{R}^{14} \cdot \Sigma\right)+\Pi_{L R}^{(2)}\left(\Sigma^{T} \cdot \bar{Q}_{L}^{14} \cdot \Sigma\right)\left(\Sigma^{T} \cdot T_{R}^{14} \cdot \Sigma\right)+\text { h.c. }, \\
& =\left(\Pi_{L R}^{(1)}+\Pi_{L R}^{(2)} s_{h}^{2}\right) s_{h} c_{h} \bar{t}_{L} t_{R}+\text { h.c. }
\end{aligned}
$$

In $\mathrm{MCHM}_{5_{\mathrm{L}}-5_{\mathrm{R}}}$ model the modification of both $h V V$ and $t \bar{t} h$ coupling depends solely on the parameter $\xi$, which leads to a strong constraint on the scale of compositeness. Using LHC data on the Higgs coupling measurements [10], the limit on $f$ at 95\% CL is found to be around $1 \mathrm{TeV}$. On the contrary, in $\mathrm{MCHM}_{14_{\mathrm{L}}-14_{\mathrm{R}}}, \Delta$ appearing in the $t \bar{t} h$ coupling (see Eq. (1.9)) depends on the masses and decay constants of the resonances [11], as the form factors cannot be totally absorbed in $m_{t}$ because of the presence of more than one Yukawa invariants. This de-correlates $h V V$ and $t \bar{t} h$ couplings resulting in a relaxation on the constraints on $f$, which is found to be around $f \geq 640$ $\mathrm{GeV}$ using LHC data. Notably, if either of $t_{L}$ or $t_{R}$ is embedded in the 14, two Yukawa invariants can be constructed.

Till now we focused on the Yukawa part of the Lagrangian involving a left- handed and a righthanded top. However, the most general effective Lagrangian involving the top quark also involves Higgs insertions in the kinetic terms and is given by

$$
\mathscr{L}=\bar{t}_{L} \phi \Pi_{L}\left(q^{2}, h\right) t_{L}+\bar{t}_{R} \phi \Pi_{R}\left(q^{2}, h\right) t_{R}+\bar{t}_{L} \Pi_{L R}\left(q^{2}, h\right) t_{R}+\text { h.c. }
$$

Notice that in the above Lagrangian we have absorbed the functional dependence on the Higgs inside the form factors for notational convenience. The top quark mass and the Yukawa coupling modifier are given as

$$
m_{t}=\left.\frac{\left|\Pi_{L R}\left(q^{2}, h\right)\right|}{\sqrt{\Pi_{L}\left(q^{2}, h\right) \Pi_{R}\left(q^{2}, h\right)}}\right|_{q^{0} \rightarrow 0, h \rightarrow v}, \quad k_{t \bar{t} h}=\frac{Y_{t}}{Y_{t}^{S M}}=\frac{1}{Y_{t}^{S M}}\left(1-\frac{\xi}{2}\right) \frac{\partial m_{t}}{\partial v} .
$$

while in the effective $g g h$ coupling modifier, the contribution from kinetic terms is absent (shown in $[13,12])$. It is given by

$$
k_{g g h}^{(t)}=\frac{g_{g g h}}{g_{g g h}^{S M}}=\left.\frac{1}{g_{g g h}^{S M}}\left(1-\frac{\xi}{2}\right) \frac{\partial \log \left|\Pi_{L R}\left(q^{2}, h\right)\right|}{\partial h}\right|_{q^{2} \rightarrow 0, h \rightarrow v} .
$$

In Fig. 1, regions allowed at $68 \%$ and $95 \%$ CL by the LHC Run-1 and Run-2 data in the $k_{t \bar{t} h}-\xi$ 

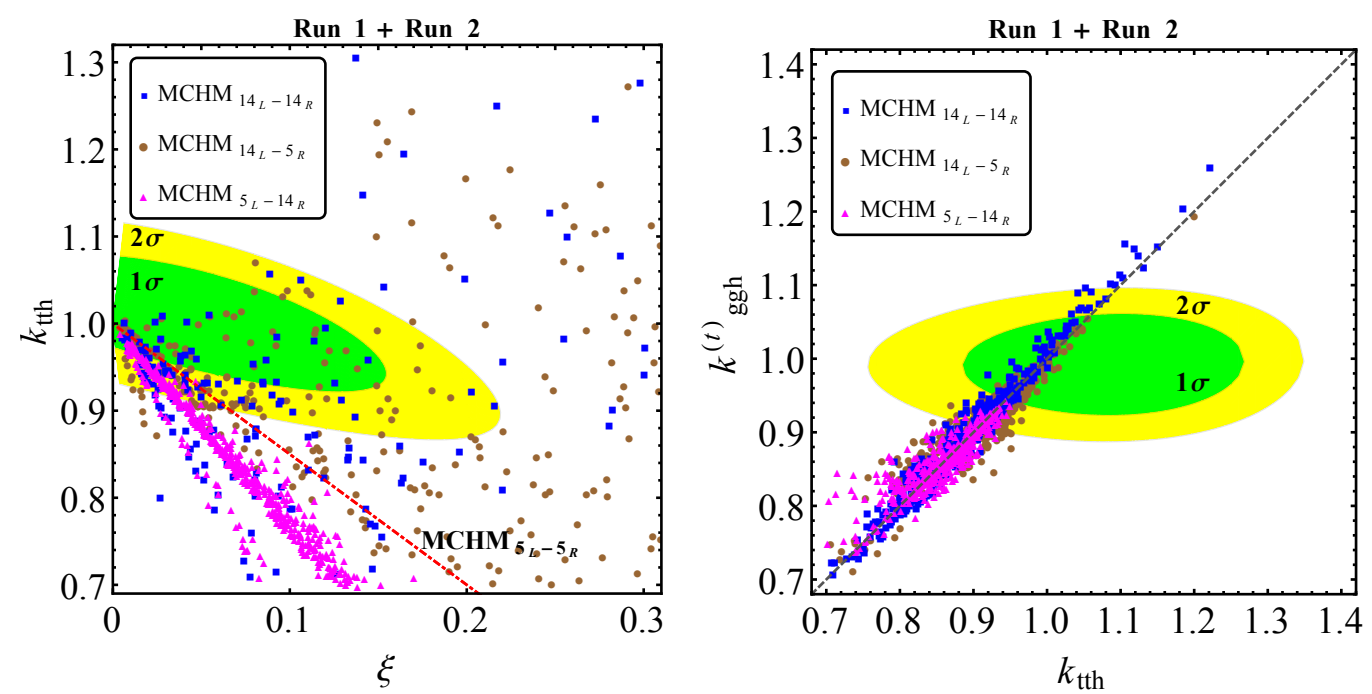

Figure 1: Ranges allowed at 68\% (green) and 95\% (yellow) CL from the LHC Run-1 and Run-2 data in the $k_{t \bar{t} h}-\xi\left(\right.$ left panel) and $k_{g g h}^{(t)}-k_{i t h}$ (right panel) planes, with scattered model points evaluated for different extended representations superimposed, adapted from [11].

and $k_{t \bar{t} h}-k_{g g h}^{(t)}$ planes are displayed. Model points for different embeddings of $t_{L}$ and $t_{R}$ are also shown on the same plots. The important point here is to note that the top quark Yukawa coupling can be larger than its corresponding SM value, in the extended representations, in contrast with the $\mathrm{MCHM}_{5_{1}-5_{\mathrm{R}}}$ model.

\section{Fine-tuning and next-to-minimal model}

As mentioned earlier, the gauge and Yukawa interactions of the Higgs explicitly break the shift symmetry of the NGBs and radiatively generates a potential for them. The explicit expression for the top quark contribution to the Coleman-Weinberg potential in case of $\mathrm{MCHM}_{5_{\mathrm{L}}-5_{\mathrm{R}}}$ is given by

$$
V_{\text {eff }}(h)=-2 N_{c} \int \frac{d^{4} q_{E}}{(2 \pi)^{4}} \log \left[-q_{E}^{2}\left(\Pi_{L}^{0}+\frac{\Pi_{L}^{1}}{2} s_{h}^{2}\right)\left(\Pi_{R}^{0}+\Pi_{R}^{1} c_{h}^{2}\right)-\frac{\left|\Pi_{L R}\right|^{2}}{2} s_{h}^{2} c_{h}^{2}\right],
$$

where $N_{c}$ denotes the number of colors of the top quark. Similarly one can calculate the contributions of the gauge boson loops to the Coleman-Weinberg potential of the pNGBs. It is worth noting that the gauge contribution alone cannot induce electroweak symmetry breaking. However, it plays an important role in tuning the electroweak vev to $246 \mathrm{GeV}$ by some cancellation with the corresponding fermionic contribution. The Higgs potential can be parametrized as

$$
V_{\mathrm{eff}}(h)=-\alpha f^{2} s_{h}^{2}+\beta f^{2} s_{h}^{4}, \quad \text { and } \quad \xi=\frac{\alpha}{2 \beta} .
$$

Note that in the above equation the coefficients $\alpha$ and $\beta$ depend on the structure of the form factors. A minimal amount of fine tuning is necessary in all composite Higgs models to justify the separation between weak scale and the scale of compositeness. This minimal tuning can be parametrized as $1 / \xi$ [14]. However, apart from the minimal vev tuning, the Higgs mass also 


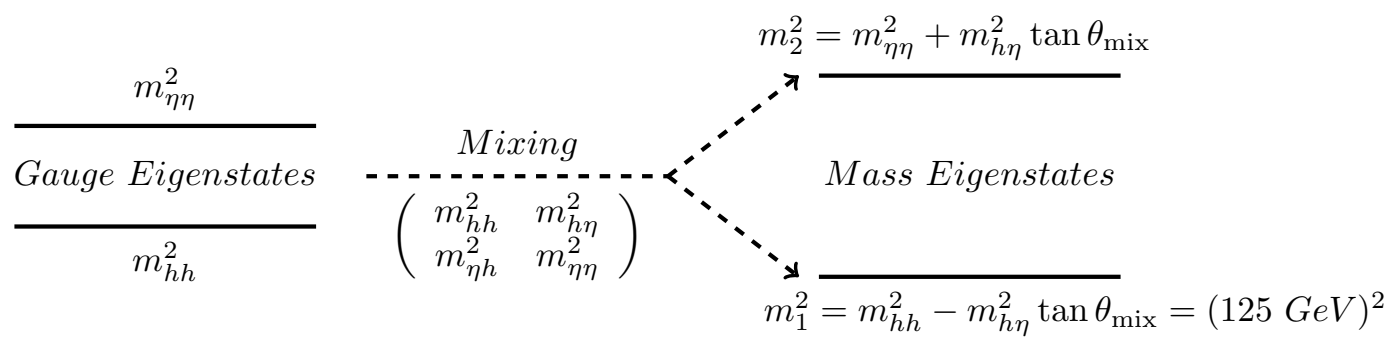

Figure 2: A schematic diagram describing level-repulsion due to mass-mixing to generate a lighter Higgs, adapted from [16].

requires further amount of fine tuning. The generic expectation for the dependence of the Higgs mass on various parameters of the composite Higgs models can be written as [15]

$$
m_{h}^{2} \sim \frac{N_{c}}{8 \pi^{2}} \frac{m_{t}^{2} m_{Q}^{2}}{f^{2}}
$$

where $m_{Q}$ denotes the mass of the lightest resonance (also called top-partner) in the strong sector. The masses of the composite resonances are naturally expected to be around $4 \pi f$. Indeed, to reproduce the correct value of the Higgs mass either large value of $f$ or smaller $m_{Q}$ is needed. While lighter resonances are heavily constrained from the direct searches at LHC, increasing $f$ amounts to the introduction of larger fine-tuning, leading to a tension in parameter space of the minimal composite Higgs model. This connection between a light Higgs and a light top-partner leading to the Higgs mass tuning can be alleviated in the next-to-minimal composite Higgs model, which we discuss below [16].

The next-to-minimal composite Higgs model, having coset $\mathrm{SO}(6) / \mathrm{SO}(5)$, produces five NGBs, among which four constitute a Higgs doublet while the remaining one is a singlet under the SM gauge group [17]. If this singlet develops a vev after the electroweak symmetry breaking, a massmixing between the neutral component of the doublet and this singlet is possible. Note that the vev and hence the mass of the singlet is expected to be around $f$ in absence of any additional tuning. The singlet, being heavier, leads to a level-repulsion due to the mass-mixing and pushes the dominantly doublet state down to the observed Higgs mass. However, the masses of both the doublet and the singlet prior to the mixing could be large avoiding too much fine-tuning. This mechanism as shown in Fig. 2 enables to create a substantial mass gap between the observed Higgs boson and the lightest top-partner. In Fig. 3, we display the contours of $m_{h}=125 \mathrm{GeV}$ in the plane of the two lightest top-partners. The black solid line, representing the next-to-minimal coset evidently allows more parameter space in comparison to the minimal coset, which is denoted by the red dashed line, for the same amount of minimal fine-tuning $(\xi=0.06)$ - and in this sense the next-to-minimal model is less tuned.

\section{Conclusions and outlook}

In composite Higgs scenario, unlike in supersymmetry, the 'big hierarchy' is solved by construction in the sense that the Higgs boson itself dissolves beyond the scale ( $4 \pi f \sim$ few tens of TeV) 


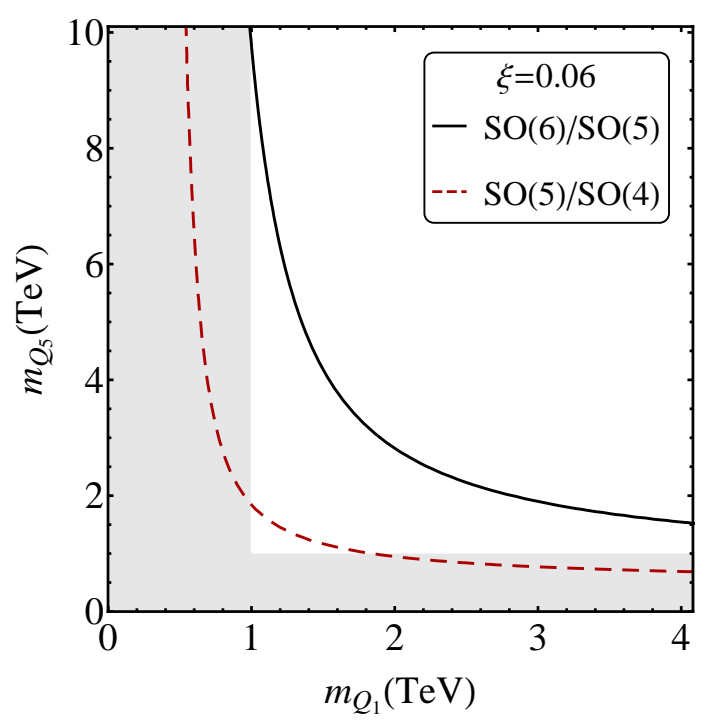

Figure 3: Contours of $m_{h}=125 \mathrm{GeV}$ for minimal (red dashed line) and next-to-minimal (black solid line) models in the plane of two lightest top-partners, gray shaded area shows the direct search constraints from LHC, adapted from [16].

of compositeness. These models interpolate between the SM $(\xi \rightarrow 0)$ and Technicolor $(\xi=1)$. They can in principle be tested at the LHC as they have verifiable predictions, namely, the Higgs couplings are modified by the pNGB dynamics. While the modifications in $h V V(V=W, Z)$ couplings are universal, modifications in Yukawa couplings depend on the representations in which the SM fermions are put under the global group $G$. While in the minimal $\mathrm{MCHM}_{5_{L}-5_{R}}$ model the constraint on the decay constant is $f>1 \mathrm{TeV}$, it is relaxed to $640 \mathrm{GeV}$ when fermions (more specifically, the top and bottom quarks) are put in extended representations. The constraint from the oblique electroweak $S$ parameter is also in the same ballpark: $f>1 \mathrm{TeV}$, modulo certain assumptions. Fine-tuning in the Higgs mass is usually a few percent in a general class of such models, but it can be somewhat relaxed in the next-to-minimal models.

\section{Acknowledgments}

This talk is based on work done with Avik Banerjee, Nilanjana Kumar and Tirtha Sankar Ray, and I thank them for sharing their insights with me, and especially $\mathrm{AB}$ for helping me during the preparation of this write-up. I acknowledge support of the J.C. Bose National Fellowship from the Department of Science and Technology, Government of India (SERB Grant No. SB/S2/JCB062/2016). Finally, I thank the Organizers of Corfu2018 for the invitation.

\section{References}

[1] D. B. Kaplan and H. Georgi, $S U(2) x U(1)$ Breaking by Vacuum Misalignment, Phys. Lett. B136 (1984) 183-186. 
[2] M. J. Dugan, H. Georgi and D. B. Kaplan, Anatomy of a Composite Higgs Model, Nucl. Phys. B254 (1985) 299-326.

[3] R. Contino, The Higgs as a Composite Nambu-Goldstone Boson, in Physics of the large and the small, TASI 09, proceedings of the Theoretical Advanced Study Institute in Elementary Particle Physics, Boulder, Colorado, USA, 1-26 June 2009, pp. 235-306, 2011. 1005 . 4269. DOI.

[4] G. Panico and A. Wulzer, The Composite Nambu-Goldstone Higgs, Lect. Notes Phys. 913 (2016) pp.1-316, [1506.01961].

[5] C. Csaki and P. Tanedo, Beyond the Standard Model, in Proceedings, 2013 European School of High-Energy Physics (ESHEP 2013): Paradfurdo, Hungary, June 5-18, 2013, pp. 169-268, 2015. 1602.04228 . DOI.

[6] G. F. Giudice, C. Grojean, A. Pomarol and R. Rattazzi, The Strongly-Interacting Light Higgs, JHEP 06 (2007) 045, [hep-ph/ 0703164$].$

[7] K. Agashe and R. Contino, The Minimal composite Higgs model and electroweak precision tests, Nucl. Phys. B742 (2006) 59, [hep-ph/0510164].

[8] K. Agashe, R. Contino, L. Da Rold and A. Pomarol, A Custodial symmetry for Zbb, Phys. Lett. B641 (2006) 62, [hep-ph/ 0605341$].$

[9] K. Agashe, R. Contino and A. Pomarol, The Minimal composite Higgs model, Nucl. Phys. B719 (2005) 165-187, [hep-ph/ 0412089$].$

[10] ATLAS, CMS collaboration, G. Aad et al., Measurements of the Higgs boson production and decay rates and constraints on its couplings from a combined ATLAS and CMS analysis of the LHC pp collision data at $\sqrt{s}=7$ and 8 TeV, JHEP 08 (2016) 045, [1606.02266].

[11] A. Banerjee, G. Bhattacharyya, N. Kumar and T. S. Ray, Constraining Composite Higgs Models using LHC data, JHEP 1803 (2018) 062, [1712.07494].

[12] M. Montull, F. Riva, E. Salvioni and R. Torre, Higgs Couplings in Composite Models, Phys. Rev. D88 (2013) 095006, [1308.0559].

[13] A. Azatov and J. Galloway, Light Custodians and Higgs Physics in Composite Models, Phys. Rev. D85 (2012) 055013, [1110.5646].

[14] G. Panico, M. Redi, A. Tesi and A. Wulzer, On the Tuning and the Mass of the Composite Higgs, JHEP 03 (2013) 051, [1210. 7114].

[15] A. Pomarol and F. Riva, The Composite Higgs and Light Resonance Connection, JHEP 08 (2012) 135, [1205.6434].

[16] A. Banerjee, G. Bhattacharyya and T. S. Ray, Improving Fine-tuning in Composite Higgs Models, Phys. Rev. D96 (2017) 035040, [1703.08011].

[17] B. Gripaios, A. Pomarol, F. Riva and J. Serra, Beyond the Minimal Composite Higgs Model, JHEP 04 (2009) 070, [0 902 . 1483]. 